\title{
Two New Topologically Ordered Glass Phases of Smectics Confined in Anisotropic Random Media
}

\author{
Brad Jacobsen, ${ }^{1}$ Karl Saunders, ${ }^{2}$ Leo Radzihovsky, ${ }^{1}$ and John Toner $^{2}$ \\ ${ }^{1}$ Department of Physics, University of Colorado, Boulder, Colorado 80309 \\ ${ }^{2}$ Department of Physics, Materials Science Institute, and Institute of Theoretical Science, University of Oregon, \\ Eugene, Oregon 97403
}

(Received 18 March 1999)

\begin{abstract}
Smectics in strained aerogel exhibit two new glassy phases. The strain both ensures the stability of these phases and determines their nature. One type of strain induces an " $X Y$ Bragg glass," while the other creates a novel, triaxially anisotropic " $m=1$ Bragg glass." The latter exhibits anomalous elasticity, characterized by exponents that we calculate to high precision. We predict the phase diagram, and numerous other experimentally observable scaling laws, for the system.
\end{abstract}

PACS numbers: 64.60.Fr, 05.40.-a, 82.65.Dp

Liquid crystals confined in random porous structures have become a subject of considerable interest [1]. A recent theoretical study unambiguously demonstrated that conventional (quasi-)long-ranged smectic order is impossible in 3D in the presence of (even arbitrarily weak) quenched pinning imposed by these random structures, e.g., aerogel [2]. It was proposed that a positionally disordered but topologically ordered "smectic Bragg glass" (SBG) phase would become the new thermodynamically distinct low-temperature phase in these smectic systems. However, for quenched random isotropic structures it was impossible to make a compelling theoretical argument for the stability of such a glass phase.

In this Letter, we make such a compelling argument for smectics in a uniaxially strained aerogel, which certainly exhibit two types of low- $T$ BG phases that are thermodynamically distinct from the high- $T$ nematic [or perhaps "nematic elastic glass" (NEG)] and isotropic liquid phases. For parallel nematogen-surface alignment (assumed throughout), a stretch (Fig. 1a) of the aerogel will lead to an " $X Y$-BG" in the isotropic universality class of randomly pinned vortex lattices, CDW's, and random field $X Y$ magnets (RF- $X Y$ ) [3], while a compression (Fig. 1b) will lead to a novel " $m=1 \mathrm{BG}$," with triaxially anisotropic scaling, that should be similar to that of a discotic in isotropic aerogel [4]. For homeotropic alignment, these two phases reverse with respect to stretch and compression, with all other results remaining unchanged.

We predict two possible low, constant- $T$ phase diagrams, depending on whether the SBG is stable (Fig. 2b)

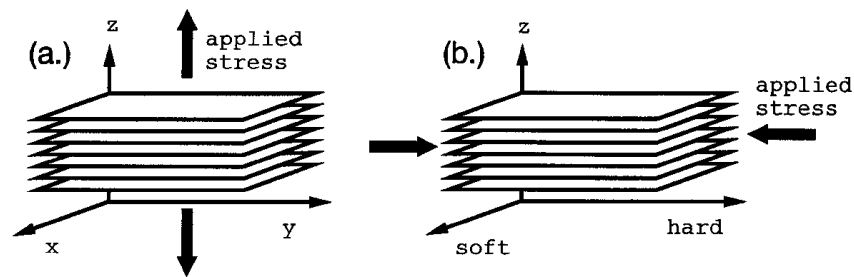

FIG. 1. (a) Stretch along the $\hat{\mathbf{z}}$ direction. (b) Compression along the $\perp$ direction. or not (Fig. 2a). Recent experiments suggest the former possibility. The loci of the phase boundaries in Fig. 2a, for small strain, $\sigma$, are universal and satisfy

$$
\Delta(\sigma) \propto\left(K^{3} B\right)^{1 / 2}(\sigma / B)^{\rho},
$$

where $\sigma$ is proportional to the uniaxial stress applied to the aerogel fibers, $\Delta$ is a measure of the tilt disorder, which in the simplest microscopic model is proportional to the aerogel density $\rho_{A}$ [2] (where the proportionality constant is an increasing function of the anchoring energy), $B$ and $K$ are bulk smectic elastic moduli, and $\rho$ is a universal exponent expressible in terms of anomalous elasticity exponents $\tilde{\eta}_{B}$ and $\tilde{\eta}_{K}$ for unstrained aerogel. Our best estimate is $\rho \approx 2 / 5$ in 3D [2].

Our model of the smectic in aerogel treats the local smectic layer displacement $u(\mathbf{r})$ and the local nematic director $\hat{\mathbf{n}}(\mathbf{r})$ as the only important fluctuating quantities, ignoring fluctuations in the magnitude $|\psi|$ of the smectic order parameter $\psi=|\psi| e^{i q_{o} u(\mathbf{r})}$ about its mean $\left|\psi_{o}\right|$.

The important effects of the aerogel are completely described by only two disorder types. One is the random-field translational disorder $\delta H_{r f}=\operatorname{Re} \int d^{d} \mathbf{r} \times$ $\left|\psi_{o}\right| V(\mathbf{r}) e^{i q_{o} u(\mathbf{r})}$, coupling to $u(\mathbf{r})$, where $V(\mathbf{r})$ is a complex random potential which at long scales can be accurately represented as zero-mean and short-ranged [2] Gaussian statistics with $V(\mathbf{r}) V^{*}\left(\mathbf{r}^{\prime}\right)=\tilde{\Delta}_{V} \delta^{d}\left(\mathbf{r}-\mathbf{r}^{\prime}\right)$. The other type of disorder is the random-tilt orientational disorder given by $\delta H_{t}=-\int d^{d} \mathbf{r}[\mathbf{g}(\mathbf{r}) \cdot \hat{\mathbf{n}}]^{2}$, describing the tendency of nematogens $\hat{\mathbf{n}}(\mathbf{r})$ to align along the local aerogel strand directed along $\mathbf{g}(\mathbf{r})$, and at long scales is
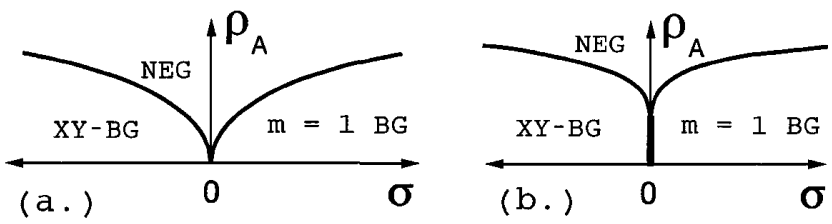

FIG. 2. Two possible phase diagram topologies, depending on whether SBG is stable for isotropic confinement $(\sigma=0)$.

(C) 1999 The American Physical Society

1363 
completely described by short-ranged [2] correlations $\overline{g_{i}(\mathbf{r}) g_{j}\left(\mathbf{r}^{\prime}\right)}=1 / 2\left(\sqrt{\Delta} \delta_{i j}-\gamma e_{i} e_{j}\right) \delta^{d}\left(\mathbf{r}-\mathbf{r}^{\prime}\right)$, where $\hat{\mathbf{e}}$ is the uniaxial direction (i.e., the axis of the strain applied to the aerogel). In the above $\tilde{\Delta}_{V}=$ $\Gamma_{u}\left(a_{f} / L_{f}\right)^{d-d_{f}}\left(1 / a_{f} q_{o}\right), \quad \Delta=\Gamma_{n}\left(a_{f} / L_{f}\right)^{d-d_{f}}, \quad d_{f}$ is the aerogel's fractal dimension for scales $a_{f}<r<L_{f}$, and $\Gamma_{u}, \Gamma_{n}, \gamma$ are phenomenological parameters, where $\gamma$ is the anisotropy parameter which at small strains is proportional to $\sqrt{\Delta}$ and the stress $\sigma$ applied to the aerogel. $\gamma<0$ for a stretch illustrated in Fig. 1a.

Assuming (as we will verify a posteriori) that fluctuations in $\hat{\mathbf{n}}$ from a perfect alignment with the smectic layer normal (taken along $\hat{\mathbf{z}}$ ) are small allows us to integrate $\hat{\mathbf{n}}$ out of the partition function, with the only effect of replacing $\delta \mathbf{n} \rightarrow \nabla_{\perp} u$ (Higg's mechanism) [2]. The resulting Hamiltonian is given by

$$
\begin{aligned}
H=\int_{\mathbf{r}} & {\left[\frac{K}{2}\left(\nabla_{\perp}^{2} u\right)^{2}+\frac{B}{2}\left(\partial_{z} u-\frac{1}{2}\left(\nabla_{\perp} u\right)^{2}\right)^{2}\right.} \\
& -\left[\mathbf{g}(\mathbf{r}) \cdot \nabla_{\perp} u\right]^{2}-2 g_{z}(\mathbf{r}) \mathbf{g}(\mathbf{r}) \cdot \nabla_{\perp} u \\
& \left.+\left[g_{z}(\mathbf{r}) \nabla_{\perp} u\right]^{2}-\left|\psi_{o}\right| \operatorname{Re}\left\{V(\mathbf{r}) e^{i q_{o} u(\mathbf{r})}\right\}\right] .
\end{aligned}
$$

The form of the anharmonic elastic terms is dictated by the underlying invariance of the bulk smectic phase under rotations about any axis lying in the $\mathbf{r}_{\perp}$ plane.

After introducing $n$ replica fields $u_{\alpha}$ and integrating out the disorder, we obtain a Hamiltonian whose form strongly depends on the type of uniaxial strain. Stretching the aerogel strands will cause the layer normal, $\hat{\mathbf{z}}$, to align with $\hat{\mathbf{e}}$ (Fig. 1a). Smectics confined inside this structure, to harmonic order in elasticity (with elastic anharmonicity irrelevant), are described by

$$
\begin{aligned}
H_{X Y}= & \frac{1}{2} \int_{\mathbf{r}} \sum_{\alpha=1}^{n}\left[K\left(\nabla_{\perp}^{2} u_{\alpha}\right)^{2}+B\left(\partial_{z} u_{\alpha}\right)^{2}+|\gamma|\left(\nabla_{\perp} u_{\alpha}\right)^{2}\right] \\
& -\frac{1}{2 T} \int_{r} \sum_{\alpha, \beta=1}^{n}\left[(\Delta+\sqrt{\Delta}|\gamma|)\left(\nabla_{\perp} u_{\alpha}\right) \cdot\left(\nabla_{\perp} u_{\beta}\right)+\Delta_{V} \cos \left[q_{o}\left(u_{\alpha}-u_{\beta}\right)\right]\right],
\end{aligned}
$$

where $\Delta_{V}=2\left|\psi_{o}\right|^{2} \tilde{\Delta}_{V}$. At scales smaller than a crossover scale $\xi_{\perp}^{c}$ (see below), the behavior is that of a smectic pinned by isotropic unstrained aerogel [2]. On longer scales, however, the scaling behavior of such an anisotropically pinned smectic crosses over to that of the RF- $X Y$ model. We therefore predict that smectics pinned by such anisotropic weak disorder will exhibit the $X Y$-BG phase, with its universal disorder-induced logarithmic layer wandering character, $\overline{\left\langle[u(\mathbf{r})-u(0)]^{2}\right\rangle}=C(d)(\ln r) / q_{o}^{2}$ [3]. However, unlike 3D bulk smectics, which show the famous Landau-Peierls thermally driven $\ln r$ fluctuations,

here $C(d)$ is universal, the logarithm persists in all $2<$ $d<4$, and smectic layers are pinned. The immediate consequence is that $\mathrm{x}$-ray scattering will exhibit real-space power-law decay $\overline{\left\langle\rho_{G}(\mathbf{r}) \rho_{-G}(\mathbf{0})\right\rangle} \propto r^{-\eta(G)}$ with a universal $\eta(G)$ exponent $\left(G=m q_{o}\right)$.

If, instead, the aerogel is uniaxially compressed, i.e., $\gamma>0$, we expect that one of the (previously soft) $\mathbf{r}_{\perp}$ smectic axes ( $x$ or $y$ ) will orient along the axis of compression $\hat{\mathbf{e}}_{h}$ (Fig. 1b). We denote this $\hat{\mathbf{e}}_{h}$-directed axis as hard $(h)$, and call the other $\perp$ axis, orthogonal to $\hat{\mathbf{e}}_{h}$, the soft $(s)$ axis, i.e., $\mathbf{r}_{\perp}=\left(\mathbf{r}_{h}, \mathbf{r}_{s}\right)$. The resulting effective Hamiltonian describing this system at long scales is

$$
H_{m=1}=\frac{1}{2} \int_{\mathbf{r}}\left[\sum_{\alpha=1}^{n} K\left(\nabla_{\perp}^{2} u_{\alpha}\right)^{2}+B\left(\partial_{z} u_{\alpha}-\frac{1}{2}\left(\nabla_{\perp} u_{\alpha}\right)^{2}\right)^{2}+\gamma\left(\nabla_{h} u_{\alpha}\right)^{2}-\sum_{\alpha, \beta=1}^{n} \frac{\Delta}{T}\left(\nabla_{\perp} u_{\alpha}\right) \cdot\left(\nabla_{\perp} u_{\beta}\right)\right],
$$

where we have neglected the positional random-field disorder, $\Delta_{V}$, which can be shown to be subdominant at long length scales [4]. $H_{m=1}$ [Eq. (4)] implies that the noninteracting propagator $G_{\alpha \beta}(\mathbf{q}) \equiv$ $V^{-1}\left\langle u_{\alpha}(\mathbf{q}) u_{\beta}(-\mathbf{q})\right\rangle_{0}=T G(\mathbf{q}) \delta_{\alpha \beta}+\Delta q_{\perp}^{2} G(\mathbf{q})^{2}$, with $G(\mathbf{q}) \equiv 1 /\left(K q_{\perp}^{4}+\gamma q_{h}^{2}+B q_{z}^{2}\right)$. As usual, at long length scales, the disorder $(\Delta)$ contribution to layer roughness dominates over the thermal $(T)$ part of $G_{\alpha \beta}(\mathbf{q})$.

We first note that for vanishing strain $\gamma \gtrsim 0$, or equivalently at very short length scales, $H_{m=1}$ and the corresponding propagator reduce to those characterizing a smectic in unstrained isotropic aerogel [2]. The asymptotic long scale behavior of the full model described by $H_{m=1}$ is reached via two independent crossovers from the Gaussian, unstrained fixed point, during which the aerogel anisotropy $\gamma$, and the nonlinear elasticity, respectively, become important. The qualitative form of this crossover is determined by the relative magnitudes of the corresponding bare couplings. For sufficiently weak strain $\left(\gamma<\gamma_{c}\right)$, the elas- tic anharmonicity becomes important first and this occurs at a crossover length scale $\tilde{\xi}_{\perp}^{N L} \propto\left(\frac{K^{5 / 2}}{B^{1 / 2} \Delta}\right)^{1 /(5-d)}$ determined by the smectic in unstrained aerogel [2]. In this case, the system first crosses over from the unstrained Gaussian to the unstrained anomalous fixed point. The final crossover to asymptotic strained anomalous behavior takes place within the anomalously elastic smectic described by the wave-vector-dependent elastic constants [2] and occurs at $\mathbf{q}_{\perp}$ such that $\tilde{K}\left(\mathbf{q}_{\perp}\right) q_{\perp}^{4} \approx \gamma q_{\perp}^{2}$, with $\tilde{K}\left(\mathbf{q}_{\perp}\right)$ calculated in Ref. [2], i.e., at $\xi_{\perp}^{c} \approx\left[K /\left[\gamma\left(\tilde{\xi}_{\perp}^{N L}\right)\right]^{\tilde{\eta}_{K}}\right]^{1 /\left(2-\tilde{\eta}_{K}\right)}$ $\left(\tilde{\eta}_{B}, \tilde{\eta}_{K}\right.$ are the exponents for isotropic disorder).

For the remainder of this paper, we will focus on the other crossover scenario in which the strain $\gamma$ is sufficiently large $\left(\gamma<\gamma_{c}\right)$ that the crossover from Gaussian unstrained to Gaussian strained elasticity takes place at $\xi_{\perp}^{c}=\sqrt{K / \gamma}$, before elastic nonlinearities become important. The critical value of $\gamma$ that delineates between these two crossover scenarios is $\gamma_{c}=K /\left(\tilde{\xi}_{\perp}^{N L}\right)^{2}$. 
For $\gamma>\gamma_{c}$, on scales longer than $\xi_{\perp}^{c}=\sqrt{K / \gamma}$ the effective Hamiltonian (and the propagator $G$ derived from it) is identical to that given in Eq. (4), but with all $\nabla_{\perp}$ replaced by $\nabla_{s}$, with $\mathbf{r}_{s}$ a subset of $\mathbf{r}_{\perp}$ axes remaining soft even in the presence of aerogel anisotropy. Our goal then is to assess the role of elastic nonlinearities, at this new strained Gaussian fixed point, which become important beyond an even longer nonlinear crossover length scale $\xi_{s}^{N L}$ (along the "soft" direction) [6].

The scale $\xi_{s}^{N L}$ can be determined from a simple perturbation theory in these nonlinear couplings of $H_{m=1}$, and is the length at which the effects of anharmonic elastic terms become significant. For example, the diagrammatic correction to the bulk modulus $B$, due to these elastic nonlinearities, is given by

$$
\begin{aligned}
\delta B(L) & =-\frac{B^{2}}{2} \int_{\mathbf{q}}^{>}\left[T G(\mathbf{q})^{2}+2 \Delta q_{s}^{2} G(\mathbf{q})^{3}\right] q_{s}^{4}, \\
& \approx \frac{-C_{d-1} \beta_{d-1}}{2 \pi(7-2 d)} \Delta\left(\frac{B^{3}}{\gamma^{d-2} K^{7-d}}\right)^{1 / 2} L^{7-2 d},
\end{aligned}
$$

where in the above we have kept only the dominant disorder-induced infrared divergent contribution, cut off these long-scale divergences by $q_{s}>1 / L$, and analytically continued to arbitrary dimension $d$, with a single smectic ordering coordinate $z$, a single soft coordinate $r_{s}$, and $d-2$ hard axes with coordinate $\mathbf{r}_{h}$. The constant $C_{d}=2 \pi^{d / 2} /\left[(2 \pi)^{d} \Gamma(d / 2)\right]$ and $\beta_{d}=\Gamma(d / 2) \Gamma(3-$ $d / 2) / 2$. For $d<d_{u c}=7 / 2$, the corrections to $B$ [Eq. (5b)] grow with cutoff $L$ and become significant for scales $L>\xi_{s}^{N L}$, such that $\left|\delta B\left(\xi_{s}^{N L}\right)\right|=B$, signaling the breakdown of conventional harmonic elasticity. We find

$$
\xi_{s}^{N L}=\left(\frac{2 \pi(7-2 d) K^{(7-d) / 2} \gamma^{(d-2) / 2}}{C_{d-1} \beta_{d-1} \Delta B^{1 / 2}}\right)^{1 /(7-2 d)} .
$$

The corresponding lengths along the $z$ and $h$ axes are given by $\xi_{z}^{N L}=\left(\xi_{s}^{N L}\right)^{2} / \lambda_{B}$ and $\xi_{h}^{N L}=\left(\xi_{s}^{N L}\right) / \lambda_{\gamma}$, where $\lambda_{B} \equiv(K / B)^{1 / 2}$ and $\lambda_{\gamma} \equiv(K / \gamma)^{1 / 2}$. Identical crossover length scales are obtained if one instead studies perturbative corrections to $K$ or $\Delta$.

To go beyond these crossover length scales $\xi_{z, h, s}^{N L}$ we use the renormalization group (RG), which consists of integrating out short-scale modes, perturbatively in elastic nonlinearities, and rescaling the lengths and long wavelength part of the fields with $r_{s}=r_{s}^{\prime} e^{\ell}, r_{h}=r_{h}^{\prime} e^{\omega_{h} \ell}, z=z^{\prime} e^{\omega_{z} \ell}$, and $u_{\alpha}(\mathbf{r})=e^{\chi \ell} u_{\alpha}^{\prime}\left(\mathbf{r}^{\prime}\right)$, so as to restore the uv cutoff back to $\Lambda \sim 1 / a$. The underlying rotational invariance ensures that the graphical corrections preserve the rotationally invariant operator $\left[\partial_{z} u_{\alpha}-\frac{1}{2}\left(\nabla_{s} u_{\alpha}\right)^{2}\right]$, renormalizing it as a whole. It is therefore convenient (but not necessary) to choose the dimensional rescaling that also preserves this operator; the appropriate choice is $\chi=2-\omega_{z}$.

Using the above-defined analytical continuation in $d$, $\mathrm{RG}$ to one-loop order, gives the following flow equations:

$$
\frac{d B}{d \ell}=\left(5+(d-2) \omega_{h}-3 \omega_{z}-\frac{3 g}{32 \sqrt{2}}\right) B,
$$

$$
\begin{aligned}
\frac{d K}{d \ell} & =\left(1+(d-2) \omega_{h}-\omega_{z}+\frac{g}{8 \sqrt{2}}\right) K, \quad(7 \mathrm{~b}) \\
\frac{d(\Delta / T)}{d \ell} & =\left(3+(d-2) \omega_{h}-\omega_{z}+\frac{g}{32 \sqrt{2}}\right)(\Delta / T),
\end{aligned}
$$

where we have defined a dimensionless measure of disorder $g=\Delta B /\left(K^{7-d} \gamma^{d-2}\right)^{1 / 2} C_{d-1} \Lambda^{2 d-7}$, which flows according to

$$
\frac{d g(\ell)}{d \ell}=2 \epsilon g-\frac{15}{64 \sqrt{2}} g^{2},
$$

with $\epsilon \equiv 7 / 2-d$. Because all relevant anharmonic terms in $H_{m=1}$ appear with $\nabla_{s}$, there are no graphs correcting $\gamma$ and therefore no anomalous $\gamma$ elasticity to all orders. As required, the flow of $g$ is independent of the arbitrary choice of the anisotropy rescaling exponents $\omega_{h}$ and $\omega_{z}$. The growth of $g$ for $d<d_{u c}=7 / 2$ is an indication that the long-scale properties of our system, even at a finite temperature $T$, are dominated by disorder. The eventual termination of this flow at a nontrivial, glassy $T=0$ fixed point $g^{*}=\epsilon 128 \sqrt{2} / 15$, leads to strong disorder-generated power-law anomalous elasticity.

One consequence of the anomalous elasticity is that the long-scale elastic constants $K, B$, and disorder variance $\Delta$ become wave vector dependent:

$$
\begin{aligned}
& K(\mathbf{k})=K k_{s}^{--\eta_{K}} f_{K}\left(k_{h} / k_{s}^{\zeta_{h}}, k_{z} / k_{s}^{\zeta_{z}}\right), \\
& B(\mathbf{k})=B k_{s}^{\eta_{B}} f_{B}\left(k_{h} / k_{s}^{\zeta_{h}}, k_{z} / k_{s}^{\zeta_{z}}\right), \\
& \Delta(\mathbf{k})=\Delta k_{s}^{-\eta_{\Delta}} f_{\Delta}\left(k_{h} / k_{s}^{\zeta_{h}}, k_{z} / k_{s}^{\zeta_{z}}\right),
\end{aligned}
$$

$\gamma(\mathbf{k})=\gamma$, with the anisotropy exponents $\zeta_{z} \equiv 2-$ $\left(\eta_{B}+\eta_{K}\right) / 2$ and $\zeta_{h} \equiv 2-\eta_{K} / 2$. The exponents obey

$$
7-2 d+\eta_{\Delta}=\frac{\eta_{B}}{2}+\frac{7-d}{2} \eta_{K},
$$

exactly, due to the underlying exact rotational invariance of Eq. (4) about $\hat{\mathbf{e}}_{h}$. To leading order in $\epsilon=7 / 2-d$, $\eta_{K}=g^{*} / 8 \sqrt{2}=16 \epsilon / 15=8 / 15, \quad \eta_{B}=3 g^{*} / 32 \sqrt{2}=$ $12 \epsilon / 15=2 / 5$, and $\eta_{\Delta}=g^{*} / 32 \sqrt{2}=2 \epsilon / 15=2 / 15$, the last equalities holding in $d=3(\epsilon=1 / 2)$. Since $\epsilon=1 / 2$ is quite small, we expect these exponents to be quantitatively accurate.

The RG $\epsilon=7 / 2-d$ expansion treatment presented above is nicely complemented by an $\hat{\epsilon} \equiv 4-d$ expansion arising from a different analytical continuation to $d$ dimensions, in which there are $d-2$ soft coordinates $\mathbf{r}_{s}$ and only a single hard axis. The corresponding exponents are given by $\hat{\eta}_{K}=3 \hat{\epsilon} / 8=3 / 8, \hat{\eta}_{B}=3 \hat{\epsilon} / 4=3 / 4$, and $\hat{\eta}_{\Delta}=\hat{\epsilon} / 8=1 / 8$, with good agreement in $d=3$ (except for $\eta_{B}$ ) with the $\epsilon=7 / 2-d$ expansion results. The exact exponent relation for the $\hat{\epsilon}=4-d$ expansion is given by $4-d+\hat{\eta}_{\Delta}=\hat{\eta}_{B} / 2+2 \hat{\eta}_{K}$ and reassuringly agrees with Eq. (10) in $d=3$.

Further accuracy can be gained by weighted averaging of the $7 / 2-d$ and $4-d$ expansions, according to $\eta_{K, \Delta} \rightarrow\left(4 \eta_{K, \Delta}+\hat{\eta}_{K, \Delta}^{s}\right) / 5$. The factor of 4 reflects the higher accuracy of the $7 / 2-d=\epsilon$ expansion. The prediction for $\eta_{B}$ is then made using the exact scaling relation, giving, in $d=3, \eta_{K}=0.50, \eta_{B}=0.26$, and $\eta_{\Delta}=0.13$. 
We now study the translational and orientational order in the presence of this strong disorder-driven anomalous elasticity. The former is characterized by the growth of smectic layer roughness with, e.g., $r_{s}$ :

$$
C\left(r_{s}\right) \equiv \overline{\left\langle\left[u\left(r_{s}, 0_{h}, 0_{z}\right)-u\left(0_{s}, 0_{h}, 0_{z}\right)\right]^{2}\right\rangle}=\int \frac{d^{3} k}{(2 \pi)^{3}} 2\left[1-\cos \left(k_{s} r_{s}\right)\right] \Delta(\mathbf{k}) k_{\perp}^{2} G^{2}(\mathbf{k}),
$$

from which the translational correlation length $\xi_{s}^{X}$ (the inverse of the x-ray diffraction peak width), can be computed via the condition $C\left(r_{s}=\xi_{s}^{X}\right) \equiv a^{2}$, where $a$ is the smectic layer spacing. $\xi_{s}^{X}$ is determined by the relative order of many crossover length scales. For $\xi_{s}^{X}<\xi_{\perp}^{c}, \xi_{s}^{X}$ is iden-

$$
\xi_{s}^{X}= \begin{cases}\xi_{s}^{N L}\left(\frac{a}{\lambda_{B}}\right)^{2}, & \xi_{\perp}^{c}<\xi_{s}^{X}<\xi_{s}^{N L}, \gamma>\gamma_{c} \\ \xi_{s}^{N L}\left(\frac{a}{\lambda_{B}}\right)^{2 /\left(\eta_{B}+\eta_{K}\right)}, & \xi_{\perp}^{c}<\xi_{s}^{N L}<\xi_{s}^{X}, \gamma>\gamma_{c} \\ \xi_{\perp}^{c}\left(\frac{a}{\lambda_{B}}\right)^{2 /\left(\eta_{B}+\eta_{K}\right)}\left(\xi_{\perp}^{c} / \tilde{\xi}_{\perp}^{N L}\right)^{\left(\tilde{\eta}_{B}+\tilde{\eta}_{K}\right) /\left(\eta_{B}+\eta_{K}\right),} & \tilde{\xi}_{\perp}^{N L}<\xi_{\perp}^{c}<\xi_{s}^{X}, \gamma<\gamma_{c} .\end{cases}
$$

tical to that due to isotropic disorder [2]. In the opposite regime, $\xi_{s}^{X}>\xi_{\perp}^{c}$, there are three possibilities, depending on whether the anomalous elasticity sets in before or after the layer roughness reaches $a$, and whether the isotropicto-anisotropic crossover takes place near the harmonic or the anomalous elastic fixed point:
From these correlation lengths, we see that it is the ratio $a / \lambda_{B}$ which determines whether $\xi_{s}^{X}$ lies in a length scale regime in which anharmonic effects are important. For small $B, \lambda_{B} \gg a$ and anharmonic effects are unimportant. Note also that, in the strained length scale regime, $\xi_{s}^{X}$ will depend on $B, K, \Delta$, and $\gamma$. Thus, one could test the predictions of Eq. (12) by measuring the dependence of $\xi_{s}^{X}$ on the strength of compression (i.e., $\gamma$ ), which could be adjusted directly. In all length scale regimes, the $\mathrm{x}$-ray correlation length is finite even as $T \rightarrow 0$, signaling the destruction of the conventional (quasi-)long-ranged translational smectic order.

As emphasized in Ref. [2], this lack of translational order does not imply that the low-temperature phase replacing the smectic is simply nematic (or isotropic). Our detailed calculations [4] indicate that, in fact, despite the lack of the (quasi-)long-ranged smectic order dislocation loops remain bound for weak anisotropic disorder, and therefore the low-temperature phase replacing the smectic must be distinct from the nematic, separated from it by a thermodynamically sharp dislocation unbinding phase transition. We call this low-temperature phase the " $m=1$ Bragg glass."

The stability of this exotic glass phase is contingent upon our implicit assumption of long-ranged orientational (nematic) order. That this assumption is valid can be easily seen by computing $\overline{\left\langle|\delta \mathbf{n}|^{2}\right\rangle}=\overline{\left\langle|\nabla u|^{2}\right\rangle}$, and taking into account the wave-vector-dependent elastic moduli $K(\mathbf{k})$ and $B(\mathbf{k})$ and disorder variance $\Delta(\mathbf{k})$, as given by Eqs. (9). There are unstrained and strained contributions to $\overline{\left\langle|\delta \mathbf{n}|^{2}\right\rangle}$, arising from modes with $q_{\perp}>1 / \xi_{\perp}^{c}$ and $q_{\perp}<1 / \xi_{\perp}^{c}$, respectively. Using the corresponding anomalous exponents $\eta_{K}, \eta_{B}, \eta_{\Delta}$ [2], in the computation of the strained and unstrained parts, for finite strain $\gamma$ and weak disorder $\Delta$, we indeed find long-ranged orientational order. In the weak strain limit $\left(\gamma<\gamma_{c}\right)$, the unstrained part dominates, in 3D growing in a universal way with decreasing strain $\gamma$ and increasing disorder $\Delta$ as $\left(\Delta^{\mu} / \gamma^{\mu-1}\right)$, where $\mu=\tilde{\eta}_{B} /\left(2-\tilde{\eta}_{K}\right)$. Using Ref. [2], we estimate $\mu$ to be $3 / 2$. Since $\overline{\left\langle|\delta \mathbf{n}|^{2}\right\rangle}$ can therefore get arbitrarily large at small $\gamma$ and large $\Delta$, we expect our system to be in the orientationally disordered liquid phase in this range of parameters. On the other hand, for large $\gamma$ and small $\Delta$ the system will exhibit long-ranged orientational order and, as illustrated in Fig. 2a, will therefore be in the $m=1$ BG phase. In analogy with the Lindemann criterion for melting, the phase boundary is roughly determined by the condition $\overline{\left\langle|\delta \mathbf{n}|^{2}\right\rangle} \approx O(1)$. This leads to the phase boundary quoted in Eq. (1), and illustrated in Fig. 2a.

On the other hand, rather than rely on the untrustworthy (in 3D) $5-\epsilon$ expansion, which predicts no SBG for isotropic $(\sigma=0)$ disorder [2], we can infer the topology of the phase diagram based on the preliminary experimental evidence [5], which suggests the stability of SBG for weak isotropic disorder. This suggests that the $m=1 \mathrm{BG}$ extends all the way down to vanishing strain, $\sigma>0$, as illustrated in Fig. $2 b$.

Light scattering, which measures director correlation $\overline{\left\langle\delta n_{i}(\mathbf{q}) \delta n_{j}(-\mathbf{q})\right\rangle}$ provides an independent means to test the predictions of the theory. Finally, since anomalous elasticity also implies a nonlinear stress-strain relation at arbitrarily weak stress, our predictions for it can be independently probed in an ac acoustic experiment, searching for an unusually large second harmonic response.

L. R. and B. J. acknowledge support by the NSF DMR9625111, the MRSEC DMR-9809555, and the A.P. Sloan and David and Lucile Packard Foundations. J. T. and K. S. were supported by the NSF DMR-9634596.

[1] Liquid Crystals in Complex Geometries, edited by G.P. Crawford et al. (Taylor \& Francis, London, 1996).

[2] L. Radzihovsky and J. Toner, Phys. Rev. B 60, 206 (1999); Phys. Rev. Lett. 78, 4414 (1997); 79, 4214 (1997).

[3] T. Giamachi and P. Le Doussal, Phys. Rev. Lett. 72, 1530 (1994); D. S. Fisher, Phys. Rev. Lett. 78, 1964 (1997).

[4] K. Saunders et al. (unpublished).

[5] T. Bellini et al. (unpublished); G. S. Iannacchione et al., Phys. Rev. E 58, 5966 (1998).

[6] G. Grinstein and R. A. Pelcovits, Phys. Rev. Lett. 47, 856 (1981); L. Golubovic et al., ibid. 69, 2535 (1992). 